\title{
Abstracts XXXIX Congresso Nazionale SIAN ITALIA Malattia Renale Cronica (MRC): consapevolezza e competenze infermieristiche dopo un anno di pandemia da SARS-COV2 Bologna 3-4 Ottobre 2021
}

\section{ACCESSI VASCOLARI}

\section{ID-01}

RISOLUZIONE PRECOCE DELLE PROBLEMATICHE FAV-RELATE: TRIAGE E TEAM WORKING

J. Bartoccini, D. Lucaroni

A.O. di Perugia, Ambulatorio di Emodialisi, Perugia (PG)

Introduzione: I problemi legati alla continuità assistenziale nel paziente con IRC legati all'accesso vascolare devono trovare al più presto una rapida soluzione per non creare momenti di vuoto nell'assistenza. L'intervento che andremo ad attuare sarà quello di un triage della FAV (fistola artero-venosa) tramite una scheda appositamente studiata, per individuare precocemente problematiche che potrebbero inficiare la vita del paziente e le prestazioni dialitiche, portando, di conseguenza, a un aumento della spesa sanitaria. L'attività dell'infermiere di famiglia sul territorio è una grande opportunità nella gestione del triage della FAV, poiché, opportunamente formato, avrà quelle abilità tecniche, relazionali, educative e collaborative che potranno aiutare nella gestione e nella prevenzione degli eventi avversi.

Materiali e Metodi: Le Linee Guida raccomandano che il monitoraggio e la sorveglianza dell'accesso vascolare facciano parte della cura fornita ai pazienti con IRC allo scopo di identificare i problemi e di intervenire precocemente per prolungare la pervietà e la sopravvivenza dell'accesso. Monitoraggio e sorveglianza sono complementari e vanno sempre combinati. Le disfunzioni della FAV primaria connesse a scarso sviluppo e maturazione, così come le disfunzioni e le complicazioni associate alla pervietà e alla funzionalità della FAV, devono essere segnalate assegnando un codice colore per identificarne la priorità di intervento. Successivamente, basandoci sulle diagnosi infermieristiche Nanda NIC (Nursing Intervention Classification) e NOC (Nursing Outcomes Classification), possiamo evidenziare le problematiche e gli interventi successivi da adottare per migliorare l'assistenza alla persona.

Risultati: La sperimentazione è avviata e i risultati inerenti alle complicanze sono ottimi.

Conclusioni: Le problematiche inerenti al primo accesso vascolare sono alte, inoltre l'incidenza sempre più elevata della popolazione che entra in dialisi determinerà la crescita degli accessi vascolari e, di conseguenza, un aumento preponderante delle problematiche con conseguente aumento della spesa sanitaria . Questo project work cerca di dare un suo punto di vista nell'ottica del welfare di oggi e di domani, proponendo un cambiamento dal punto di vista socio culturale per fare fronte alle problematiche emergenti nel nuovo mercato sanitario. In quest'ottica, è di fondamentale importanza l'implementazione di un sistema di triage degli accessi vascolari da parte dell'infermiere di famiglia e del personale dei Centri Dialisi.

\section{ID-24}

I SISTEMI PER IL MONITORAGGIO DEL KT/V DELL'ACCESSO

VASCOLARE: UTILITÀ PRATICA

D.C. Massarenti, S. Cappelletti

U.O. Dialisi Asst Lariana San Fermo della Battaglia, Como (CO)

Introduzione: Numerosi studi hanno dimostrato l'esistenza di una correlazione, nel paziente in terapia sostitutiva, tra dose dialitica somministrata e mortalità e morbilità dei pazienti. Ne consegue che, per evitare gli effetti negativi, talora irreversibili, della dialisi inadeguata è indispensabile il routinario monitoraggio della dose dialitica somministrata. II KT/V, vale a dire la clearance frazionale dell'urea, assunta quale marker della tossicità uremica, è, attualmente, l'indice di più esteso utilizzo per la prescrizione e la quantificazione della dose dialitica somministrata. Per ogni incremento di 0,1 del KT/V è stata dimostrata una correlazione tra mortalità e morbilità dei pazienti in terapia sostitutiva e apporto proteico. II gruppo medico e infermieristico, al fine di garantire l'adeguatezza del trattamento, ha deciso di verificare l'entità dell'apporto dietetico di proteine che, nel soggetto in steady-state, è equivalente alla quota catabolizzata e comunemente definito il Protein Catabolic Rate (PCR).

Materiali e Metodi: 37 pazienti (20 M - 61 \pm 8 anni) portatori di protesi vascolare all'arto superiore, sottoposti a bicarbonato-dialisi trisettimanale (monitor Fresenius 5008), sono stati sottoposti, nei primi 90' della seduta dialitica, a misurazione del ricircolo (\%) e della portata (QA) $(\mathrm{mL} / \mathrm{min})$ dell'accesso protesico con Transonic ${ }^{\circledast}$ e, successivamente, a misurazione del ricircolo e del $\mathrm{Qb}$ effettivo a linee nella corretta posizione e a linee invertite con ausilio Twister-Fresenius. Sono state eseguite due serie di misurazioni per singolo metodo durante la stessa seduta. I dati sono stati utilizzati per il calcolo della portata secondo la formula di Krivitsky, Mercadal, Schneditz e Wijnen. I dati sono stati analizzati per determinare un coefficiente di correlazione.

Risultati: I risultati ottenuti evidenziano la regressione lineare $r 0,9131 p<$ 0,0001 della misurazione effettuata con Transonic ${ }^{\circledR}$ (pTra) e quella derivata dalla formula di Krivitsky (pKri): è, quindi, possibile scrivere la seguente formula:

ptran $=1,2864 *$ pKri-20,3237

Le correlazioni tra le formula di Mercadal, Schneditz e Wijinen presentano una regressione polinominale, con distorsione per valori di portata elevati, mentre, nel range di monitoraggio, la relazione si presenta lineare.

II corretto monitoraggio dell'accesso vascolare può essere effettuato utilizzando i metodi indiretti che presentano un'alta correlazione con le misurazioni ottenute da Transonic ${ }^{\circledast}$, applicando, poi, delle semplici formule matematiche.

Abbiamo implementato il nostro software di registrazione, per cui, registrando i valori del QB e dei ricircoli a linee dritte e invertite, si ottiene la QA-BTM, ricontrollata, poi, con metodo Transonic ${ }^{\circledR}$ e periodicamente con Doppler US. Sulla base delle nostre osservazioni, abbiamo proposto la seguente flowchart; oltre alla portata, altri parametri devono essere considerati per una visione globale, per il monitoraggio dell'accesso vascolare:

I parametri da associare al flusso della FAV sono:

$\checkmark$ Indicatore di efficacia dialitica (KT/V-KT)

$\checkmark$ Valori della pressione venosa (incremento nel tempo) e della pressione arteriosa (decremento nel tempo)

$\checkmark$ Velocità della pompa sangue (QB $<250 \mathrm{~mL} / \mathrm{min})$

$\checkmark$ Difficoltà ripetute nella venipuntura

$\checkmark$ Tempo di emostasi (incremento nel tempo)

$\checkmark$ \% del ricircolo (aumento nel tempo)

$\checkmark$ Decremento della QA misurato rispetto al precedente

Conclusioni: La buona funzionalità e la pervietà dell'accesso vascolare sono componenti fondamentali per la persona in trattamento emodialitico, in quanto consentono la sua stessa sopravvivenza, dal momento che contribuiscono alla buona riuscita della dialisi.

Il tempo è fondamentale nella rilevazione delle complicazioni dell'accesso vascolare. La diagnosi precoce permette di evitare il ricovero del paziente o di mettere in atto azioni correttive (PTA protesica), che possono impedire il fallimento finale dell'accesso vascolare, evitando, così, procedure urgenti, il posizionamento di un catetere venoso centrale o periferico o, nei peggiori dei casi, la sostituzione dell'accesso stesso.

Al fine di rendere la sorveglianza delle FAV P condivisa da tutto il personale infermieristico e medico, abbiamo creato un sistema di registrazione elettronica dei dati all'interno di una documentazione preposta e accessibile a tutte le figure professionali (Gepadial), dove, ad ogni seduta dialitica, vengono riportati i valori delle pressioni di aspirazione e di rientro, il valore del ricircolo, il KT/V raggiunto e, mensilmente, i valori della portata calcolata con metodo "BTM" II corretto monitoraggio dell'accesso vascolare può essere effettuato utilizzando i metodi indiretti che presentano un'alta correlazione con le misurazioni ottenute da Transonic ${ }^{\circledR}$. 


\section{MODELLI ORGANIZZATIVI}

\section{ID-08}

ESPERIENZA DI FORMAZIONE BLENDED: ECOGRAFIA INFERMIERISTICA PER INCANNULAZIONE E GESTIONE DELLA FISTOLA ARTERO-VENOSA F. D'Achille ${ }^{1}$, N. Fanano ${ }^{1}$, A. Moreci ${ }^{1}$, A. Casagrande ${ }^{1}$, T. Bartolini ${ }^{1}$, C. Migotto ${ }^{1}$, C. Mamone ${ }^{1}$, D. Franciosi ${ }^{1}$, E. Pucci ${ }^{1}$, G. D'Onorio ${ }^{1}$, I. Dargento ${ }^{1}$, M. Pendenza ${ }^{1}$, P. Pellegrino ${ }^{1}$, S. Mastrantonio ${ }^{1}$, S. Biondi ${ }^{1}$, V. Caracciolo ${ }^{1}$, A.R. Di Massimo', L. Pettorini' ${ }^{2}$ L. Di Meo ${ }^{3}$

${ }^{1}$ UOC Professioni Infermieristiche: UOCA Nefrologia e Dialisi della Asl Roma 6, Ariccia-Anzio (RM)

${ }^{2}$ UOCA Nefrologia e dialisi Asl Roma 6, Ariccia (RM)

${ }^{3}$ UOC Professioni Infermieristiche: Ufficio Infermieristico Distretto H5 ASL Roma 6, Velletri (RM)

Introduzione: L'uso dell'ecografia per gli infermieri in emodialisi è di supporto all'assistenza, in quanto permette di guidare l'operatore nella procedura di puntura della Fistola Artero-Venosa (FAV). L'esigenza di formare gli infermieri in dialisi sulla puntura eco-guidata della FAV nasce dalla necessità di approcciare il paziente portatore di FAV difficile.

Materiali e metodi: 17 infermieri in servizio presso l'UOCA Nefrologia e Dialisi dell'Ospedale dei Castelli, Ariccia (RM), sono stati inseriti in un programma di formazione Blended in due edizioni, dal 23.09.2019 al 13.12.2019 e dal 24.09.2019 al 13.12.2019. Dei 17 infermieri, 3 avevano una formazione pregressa nell'ambito dell'ecografia infermieristica attraverso l'acquisizione di crediti ECM specifici, patrocinati da EDTNA e/o aziendali. Ogni edizione del corso è stata strutturata da un evento formativo residenziale di 6 ore e 20 ore di formazione sul campo per discente. La prima edizione è stata rivolta ad un gruppo di nove discenti; la seconda, ad un gruppo di 8 discenti. Dal 25.09.2019 al 13.12.2019, i discenti hanno effettuato formazione sul campo (FsC) di 20 ore cadauno, per un totale complessivo di ore 340 di FsC. La parte pratica ha previsto un affiancamento con il tutor con rapporto 1/1: ciò ha richiesto un periodo di tempo di tre mesi. I due tutor sono dirigenti medici di $1^{\circ}$ livello, in possesso di certificazione SIUMB per ecografia nefrologica, con competenze specifiche sugli accessi vascolari. Gli infermieri sono stati valutati con esame pratico riportato su scheda contenente 10 item, ogni item descrive le skills acquisite valutate su una scala Likert da 1 a 5 . Il valore minimo da raggiungere corrisponde a 24, il massimo a 50. Dal 14.12.2019, per 60 giorni si è valutata la ricaduta dell'evento formativo su campo ovvero, la gestione infermieristica e la venipuntura della FAV eco-guidata, in autonomia; i dati raccolti sono stati documentati in cartella clinica e trascritti su scheda per il riepilogo.

Risultati: A fine del corso gli infermieri hanno raggiunto gli obiettivi proposti: acquisizione della competenza infermieristica nella incannulazione e gestione della FAV; appropriatezza delle prestazioni sanitarie; acquisizione dei principi e delle procedure EBN. Dal 14.12.2019 ad oggi l'ecografo è stato utilizzato dagli infermieri per: $22 \%$ per pungere la FAV, $11 \%$ sia per pungere che per valutare FAV, il $67 \%$ per valutare la FAV. La maggiore motivazione all'utilizzo è rappresentata dalla valutazione della FAV difficile $(55 \%$, di cui il $66 \%$ in presenza di ematoma); il restante $45 \%$ è suddiviso tra: valutazione (mappatura) FAV nativa, FAV revisionata, controllo corretto posizionamento degli aghi, eccessivo valore di PV. Si descrive brevemente (quale esempio) un caso rappresaentativo: valutazione della FAV per eccessiva Pressione Venosa (>220) durante il trattamento emodialitico. L'ecografia dimostra pervietà dell'ago di rientro che però appare inserito all'interno di un processo organizzativo trombotico intravasale. Richiesto intervento del medico reperibile che prescrive terapia anticoagulante a domicilio, e si modifica il sito di puntura dell'ago venoso di rientro (a monte del processo trombotico).

Conclusioni: I dati rilevati si riferiscono all'utilizzo dell'ecografo da parte degli infermieri nei primi 60 gg dopo il corso, questi hanno evidenziato un netto miglioramento dell'assistenza al paziente emodializzato. Tuttavia, il 2020 ha modificato e rallentato il percorso in quanto l'emergenza sanitaria ha richiesto priorità assistenziali spesso non compatibili con le attività di formazione, essendo stata la dialisi dell'OdC Centro Covid di riferimento aziendale. Con la fase IX dell'emergenza sanitaria e la nuova configurazione della Rete Covid-19, il Centro dialisi dell'OdC riprende progressivamente le attività in elezione, tra cui il proseguimento della rilevazione di nuovi dati. Tuttavia, dai dati raccolti in nostro possesso, si può affermare che I'utilizzo dell'ecografo per valutare e pungere la FAV migliora l'assistenza infermieristica al paziente emodializzato ed assicura la sopravvivenza della FAV, alla quale è legata la sua qualità di vita.

\section{ID-02}

L'ORGANIZZAZIONE MODULARE PER MIGLIORARE LA PRESA IN CARICO DEL PAZIENTE NEFROPATICO: LA NOSTRA ESPERIENZA W. Longo, S. Belgio, A. Poletti, D. Rongioletti

Degenza Nefrologica, Dialisi e Ipertensione, Degenza Nefrologica, Dialisi e Ipertensione. AOU-IRCS-Policlinico S. Orsola-Malpighi, Bologna (BO)

Introduzione: La nostra unità operativa aveva al suo attivo 22 posti letto di nefrologia suddivisi in due settori ( 6 letti per acuti e 16 di degenza ordinaria); l'assistenza erogata all'utente era puramente funzionale e non permetteva una visione d'insieme delle esigenze dei pazienti; gli svantaggi di questo modello erano: perdita della visione globale della persona assistita, estrema frammentazione delle cure, approccio meccanico e impersonale, comunicazione frammentaria tra gli infermieri, impossibilità di applicare pienamente il processo di assistenza infermieristica e conseguente insoddisfazione del paziente, impossibilità di valutare i risultati dell'assistenza e scarsa integrazione tra personale medico e personale infermieristico.

Materiali e Metodi: Attraverso la presa di coscienza dei limiti che il functional nursing possedeva, abbiamo cambiato il modello organizzativo, passando a una organizzazione di tipo modulare con infermiere case manager (ICM), suddividendo il settore di degenza di 16 posti letto in due settori da 8 posti letto ciascuno. Nella nuova organizzazione con i tre settori ( 6 letti per acuti, 8 letti di degenza e altri 8 letti di degenza), abbiamo ridistribuito le presenze giornaliere nei tre turni tenendo presente il nuovo modello organizzativo e, quindi, nel settore dedicato agli acuti, sono presenti, come in passato, durante il turno di mattina, un infermiere turnista e un ICM; abbiamo eliminato la figura dell'infermiere che lavorava per compiti e, quindi, nei due settori di degenza nel turno di mattina, sono presenti un infermiere per ciascun settore e un ICM trasversale che, come per il collega dei letti per acuti, è responsabile della globalità del percorso assistenziale e governa un percorso complesso e multidisciplinare. Nel turno di pomeriggio, sono presenti un infermiere per ogni settore che assicura la continuità del progetto assistenziale in tutti $\mathrm{i}$ suoi aspetti. Di notte, le presenze infermieristiche restano 2. In questo nuovo contesto, gli operatori di supporto non sono più semplici esecutori ma sono inseriti nel gruppo di lavoro e perseguono, in stretta collaborazione con il personale infermieristico, gli obiettivi della persona assistita.

Risultati: Gli indicatori utilizzati per valutare le ripercussioni del cambiamento sulla pratica hanno riguardato in buona parte la corretta compilazione della documentazione infermieristica e, quindi, l'accertamento, la pianificazione dell'assistenza e del diario integrato e la corretta rilevazione/registrazione dei parametri vitali, del dolore e del peso; sono stati, inoltre, valutati i tempi di esecuzione delle cure igieniche. Si è osservato un miglioramento della presa in carico con maggiori attenzione e consapevolezza del percorso del paziente e una maggiore soddisfazione da parte del personale. Rimangono difficoltà legate alla documentazione infermieristica, per cui è stata fatta apposita formazione a ottobre 2019 ed è stato impostato un percorso in itinere di revisione della stessa. Attraverso il briefing giornaliero con la componente medica, abbiamo migliorato la presa in carico e la consapevolezza del percorso del paziente. Dopo la nostra esperienza di miglioramento dell'assistenza, anche i medici hanno iniziato un percorso di cambiamento organizzativo a vantaggio del paziente e della continuità assistenziale e clinica. È in fase di progettazione un nuovo corso di formazione specifico per infermieri di nefrologia.

Conclusioni: Il passaggio a un'organizzazione di tipo modulare è un grosso passaggio culturale per il personale infermieristico e ciò diventa una grande opportunità per identificare e migliorare le competenze, per migliorare l'assistenza erogata a vantaggio della continuità assistenziale e, non da meno, per facilitare l'integrazione tra i vari membri del gruppo di lavoro, in un'ottica il più possibile multidisciplinare.

\section{ID-10}

ADDESTRAMENTO INFERMIERISTICO PER UNA TERAPIA DIALITICA ADEGUATA

V.N. Lacerenza ${ }^{1}$, L. Papagni ${ }^{2}$, G. Petruzzelli ${ }^{2}$, A. Maggiulli ${ }^{2}$, M. Massaro ${ }^{3}$, M. Giannetto ${ }^{2}$, S. Di Paolo ${ }^{2}$

${ }^{1}$ U.O. Nefrologia-Dialisi Ospedale della Murgia "F. Perinei", Altamura (BA) 'U.O. Nefrologia-Dialisi Ospedale "Mons. Dimiccoli", Barletta (BT)

${ }^{3}$ Servizio 118 Ospedale "L. Bonomo", Andria (BT)

Introduzione: In seguito a una radicale evoluzione del nostro sistema sanitario nazionale, la sanità, basata da anni sul concetto di medicina di attesa, sta evolvendosi verso una nuova concezione di medicina di iniziativa. Questo studio prende in esame una delle patologie croniche più invalidanti, 
I'insufficienza renale cronica, che, nel nostro paese, colpisce all'incirca 2.200.000 persone. II progetto di ricerca si è svolto presso l'ambulatorio sperimentale nefrologico Ma.Re.A., facente parte dell'U.O. Nefrologia-Dialisi dell'Ospedale "Mons. R. Dimiccoli" di Barletta. Lo scopo di questa ricerca è stato quello di analizzare il ruolo educazionale, oltre che di coordinamento, della figura infermieristica, andando a cogliere i cambiamenti della qualità di vita dei pazienti accorsi a tale ambulatorio.

Materiali e Metodi: È stato utilizzato un questionario basato sui modelli assistenziali di Marjory Gordon, rispettivamente somministrato a t0, t1 (6 mesi) e t2 ( 1 anno). La modalità operativa adottata è stata di tipo longitudinale e si è svolta tra settembre 2017 e settembre 2018. Tale progetto pone il paziente al centro del proprio percorso terapeutico, avendo come scopo quello di ritardare l'inizio del trattamento sostitutivo e, allo stesso tempo, di condurlo a una scelta consapevole del tipo di trattamento dialitico. L'attività dell'ambulatorio è strutturata secondo un programma di incontri con cadenza mensile, ciascuno dei quali si svolge secondo un percorso predefinito. Ad ogni incontro, dal punto di vista dell'assistenza infermieristica, è stata aggiornata la cartella infermieristica, nella quale sono stati registrati l'anamnesi e gli 11 bisogni assistenziali infermieristici. In concomitanza alla valutazione clinica della funzionalità renale di ogni singolo paziente, basata sul monitoraggio di creatininemia e velocità di filtrazione glomerulare, è stato redatto un questionario utile a valutare eventuali cambiamenti nella qualità di vita dei pazienti. Il questionario è stato somministrato a 37 pazienti, divenuti 35 a causa di due decessi, in tre tempi. Questo per valutare variazioni della funzionalità renale, in risposta al continuo rimodellamento del piano terapeutico e dell'apporto di un regime dietetico aproteico.

Risultati: Analizzando i risultati, è emerso come il $68 \%$ degli utenti abbia continuato la terapia conservativa, dato molto importante, considerando la criticità della patologia. L'8\% si è sottoposto alla dialisi peritoneale, risultato che rispecchia l'efficacia dell'educazione infermieristica, vista l'iniziale ostilità da parte dei pazienti per questo tipo di trattamento che richiede maggiore compliance. II $14 \%$ ha iniziato un percorso terapeutico emodialitico, il $5 \%$ ha optato per il trapianto, mentre il restante $5 \%$ è rappresentato dai pazienti che, nel corso della sperimentazione, sono deceduti.

Conclusioni: Si evince che questo nuovo approccio al paziente, che ha posto come obiettivo quello di arrivare a una scelta consapevole e condivisa del trattamento dialitico da intraprendere, è risultato soddisfacente grazie al ruolo cruciale che ricopre la figura dell'infermiere, il quale attua interventi assistenziali di qualità che possono influenzare o ritardare il trattamento sanitario. Gli esiti ottenuti sono stati decisamente superiori alle più rosee aspettative, in quanto la qualità di vita dei 37 pazienti, basandoci sui questionari somministrati, ha riportato un evidente miglioramento psicologico e fisico del campione preso in esame.

\section{STUDI OSSERVAZIONALI}

\section{ID-19}

\section{DIFFERENZA TRA ESSERE CURATO E SENTIRSI CURATO: EFFETTO DEL} PLACEBO NELLA TERAPIA DEL DOLORE NEI PAZIENTI IN TERAPIA EMODIALITICA CRONICA

C. Mocco, M. Leoni, F. Moro, A. Marongiu, V. Gigante, M. Marongiu,

P. Putzu, B. Casu, C. Marras, M. Cadeddu, S. Murtas

S.C. Emodialisi, ASSL di Cagliari, Centro Dialisi, Muravera (CA)

Introduzione: La letteratura indica che il $37-50 \%$ dei pazienti in emodialisi presenta dolore acuto e cronico e prurito uremico. II dolore affrontato male determina conseguenze negative sulla qualità della vita del paziente e sull'accettazione della malattia. L'effetto placebo avviene attraverso un processo in cui è fondamentale la relazione medico/infermiere-paziente. Si avviano dei meccanismi di attesa e di miglioramento che attivano delle sostanze prodotte dall'encefalo che possono realmente far diminuire il dolore. Scopo di questa piccola indagine è verificare l'efficacia nel controllo del dolore durante la seduta dialitica con il placebo (2). L'utilizzo in prima istanza del placebo può contribuire, inoltre, a riconoscere la natura del dolore, vale a dire se si tratta di dolore psicologico o fisico, e a ricorrere, quindi, al trattamento farmacologico solo quando è realmente necessario.

Materiali e Metodi: Studio osservazionale della durata di 12 mesi nei pazienti che necessitavano di terapia antalgica a esclusione del dolore secondario a patologie oncologiche, ortopediche acute, sindrome ischemica arti inferiori e dolore di origine cardiaca. La metodologia prevede l'utilizzo del placebo in prima istanza somministrando sodio cloruro allo $0,9 \%$ da $50 \mathrm{ml}$ in infusione endovenosa nel pozzetto arterioso del monitor di dialisi in circa 20'. Per la raccolta dati, si è utilizzata una scheda elaborata con Excel dove l'infermiere che ha in carico il paziente dovrà indicare per ogni episodio i seguenti dati: data, dati anagrafici, sintomo, ora di dialisi di comparsa del sintomo, valutazione del dolore pre/post placebo, remissione del dolore ed eventuale impiego di terapia farmacologica per mancato effetto del placebo. Per la valutazione del dolore, è stata utilizzata la scala di Valutazione Numerica NRS. II placebo in dialisi è stato sempre usato in accordo con il medico di turno. I sintomi devono sempre essere valutati prima di somministrare qualsiasi terapia sintomatica, evitando, con la somministrazione del placebo in prima istanza, di ritardare l'utilizzo di una terapia farmacologica efficace, procurando disagio, malessere o prolungando il dolore al paziente o il periodo di permanenza in dialisi.

Risultati: Nel periodo dello studio, sono stati osservati 51 pazienti, di cui 13 sono stati considerati idonei allo studio. Si è fatto ricorso al placebo in 105 episodi dolorosi su un totale di 1.600 sedute emodialitiche, nel $63 \%$ dei casi per dolori muscolo/scheletrici e, nel restante $37 \%$, per dolore di altra natura, nell' $87 \%$ dei casi durante la $2^{\text {a }}$ e la $3^{\text {a }}$ ora di dialisi. Nel $70 \%$ dei casi, si è verificata una risoluzione totale del sintomo solo con il placebo e, nel $16 \%$ dei casi, una riduzione parziale del dolore senza necessità di un ulteriore antidolorifico. Il placebo si è dimostrato inefficace nel $14 \%$ delle somministrazioni. La somministrazione del placebo non ha compromesso in alcun modo la possibilità di utilizzare comunque un analgesico in caso di necessità, quindi è stato garantito il rispetto dell'individuo che, comunque, non è stato disatteso nei suoi bisogni né privato della possibilità di trattamento.

Conclusioni: Nella nostra piccola esperienza, l'uso del placebo si è dimostrato efficace nel controllo del dolore nei pazienti in emodialisi, riducendo il ricorso alle terapie farmacologiche. La somministrazione del placebo tutela, quindi, il paziente da un'eccessiva somministrazione di farmaci non privi di effetti collaterali ed evita il wash out dialitico del farmaco, posticipandone, qualora fosse necessaria, la somministrazione alla fine della seduta emodialitica. In realtà, non è stata omessa una terapia salvavita e in qualsiasi momento si è potuto recedere dalla scelta iniziale. La somministrazione di un non farmaco di per sé non arreca danno e gli studi indicano una percentuale di successo. II perfezionamento di queste conoscenze può migliorare i risultati delle terapie attive, con ripercussioni cliniche positive sui pazienti in emodialisi e sulla loro qualità di vita.

\section{ID-29}

\section{ADESIONE ALLA TERAPIA FARMACOLOGICA E DIETETICA: STUDIO} OSSERVAZIONALE E RETROSPETTIVO

S. Savini ${ }^{1}$, G. Quintavalle ${ }^{2}$, C. Matera ${ }^{3}$, C. Turci ${ }^{1}$, F. Arnaldi ${ }^{4}$, D. Bonini ${ }^{5}$, G. Campestre ${ }^{4}$, D. Capra ${ }^{5}$, N. Capretti 5 , S. Caputo 5 , S. Caputo 5 , R. Carlone , $^{4}$ M. Cianfrini ${ }^{5}$, C. Cornacchia ${ }^{5}$, T. D'Urzo ${ }^{5}$, M. Giordano ${ }^{5}$, G. Licciardi $^{5}$, C. Lottatori' ${ }^{5}$, R. Michel ${ }^{5}$, P. Monarca ${ }^{4}$, M. Ricci ${ }^{5}$, F. Rocchi ${ }^{5}$, E. Scarpo ${ }^{4}$,

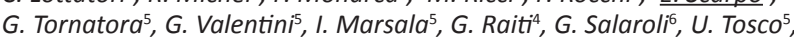
F. Flocccari ${ }^{5}$, F. Marrocco

${ }^{1}$ Dipartimento delle Professioni Sanitarie e Sociali Asl Roma 4, Civitavecchia (RM)

${ }^{2}$ Direzione Generale Asl Roma 4, Roma (RM)

${ }^{3}$ Direzione Aziendale Asl Roma 4, Roma (RM)

${ }^{4}$ Unità di Dialisi Decentrata Asl Roma 4, Bracciano (RM)

${ }^{5}$ U.O.C. Nefrologia e Dialisi Asl Roma 4, Civitavecchia (RM)

${ }^{6}$ Infermieristica Bracciano Sapienza-Asl Roma 4, Roma (RM)

Introduzione: I pazienti in dialisi sono sottoposti a forti fattori stressanti sia fisici che mentali: dolore, restrizione di liquidi, prurito, limitazione nell'attività fisica, deficit nella cura di sé, sentimenti di inadeguatezza, depressione. Il successo del trattamento dipende in larga misura dall'aderenza al regime terapeutico e alla concorrenza di competenze di diversi specialisti che intervengono sul e per il benessere del malato.

Materiali e Metodi: Gli obiettivi sono valutare l'aderenza alla terapia (dietetica e farmacologica) dei pazienti con insufficienza renale cronica sottoposti a emodialisi e migliorare le conoscenze dei pazienti per la gestione della salute. Criteri di non aderenza al regime terapeutico e ai liquidi: Potassiemia media $>6 \mathrm{mEq} / \mathrm{L}$, Fosforemia media $>5,5 \mathrm{mg} / \mathrm{dL}$, IDWG medio $>5,7 \%$ del peso corporeo "a secco". II questionario sull'adesione alla terapia farmacologica e dietetica (QAF) valuta gli atteggiamenti e le rappresentazioni del paziente in merito alla terapia farmacologica a domicilio e alla dieta. È necessario il consenso informato di tutti i pazienti.

Risultati: Il campione preso in esame comprende $\mathrm{n}^{\circ} 76$ pazienti con IRC in trattamento dialitico sostitutivo, il $34 \%$ rappresentato da donne e il $66 \%$ da uomini. Età media 70,7 anni, età dialitica media di 5,19 anni (da 4 mesi a 37 anni). Valori medi di fosforemia $>5,5 \mathrm{mg} / \mathrm{dL}$ sono stati riscontrati nel 
$35,82 \%$ dei pazienti nel totale delle 9 rilevazioni; $n^{\circ} 23$ pazienti non hanno mai superato il cut-off stabilito e $n^{\circ} 5$ hanno sempre superato il cut-off. Valori medi di potassiemia $>6 \mathrm{mEq} / \mathrm{L}$ sono stati riscontrati nel $42,63 \%$ dei pazienti nel totale delle 9 rilevazioni; $n^{\circ} 35$ pazienti non hanno mai superato il cut-off stabilito e solo uno lo ha superato sempre. Nelle 27 rilevazioni dell'IDWG > 5,7\% del peso corporeo "a secco", è risultato non aderente il $31,58 \%$ dei pazienti ( $n^{\circ} 24$ sul totale di $n^{\circ} 76$ ). Per quanto riguarda la QAF, rispetto alla fiducia nei farmaci, il $52,6 \%$ del campione $\left(n^{\circ} 40\right)$ non si dimentica di assumere i farmaci, il 73,7\% ( $\left.n^{\circ} 56\right)$ è attento alla precisione degli orari nei quali deve assumere le medicine, l' $84,2 \%$ ( $\left.n^{\circ} 64\right)$ non rifiuta le medicine prescritte e il $75 \%$ ( $n^{\circ} 57$ ) riferisce benessere all'assunzione. Per il $54 \%\left(n^{\circ} 41\right)$ del campione, assumere le medicine non fa sentire malati. Metà del campione ritiene di assumere troppi farmaci. Rispetto alla conoscenza della prescrizione terapeutica, metà del campione è attento a mangiare cibi contenenti il fosforo, il 62\% ( $\left.n^{\circ} 48\right)$ è attento ad assumere cibi con potassio, il 68\% ( $n^{\circ} 52$ ) riferisce di bere troppo e l’80,3\% ( $\left.n^{\circ} 61\right)$ sa esattamente quanto può bere al giorno. Per quanto riguarda i comportamenti corretti, il $57 \%$ del campione $\left(n^{\circ} 43\right)$ non mangia frutta e I' $80 \%\left(n^{\circ}\right.$ 60) non mangia molte verdure, il $67 \%\left(n^{\circ} 51\right)$ non consuma latte e derivati e il $51 \%\left(n^{\circ} 39\right)$ non si sente meglio quando assume le medicine. Per quanto riguarda la continuità nell'assumere i farmaci, il $96 \%$ del campione $\left(n^{\circ} 73\right)$ assume sempre le medicine, non solo quando si sente malato.

Conclusioni: Lo studio ha permesso di individuare le caratteristiche relative alla compliance terapeutica e dietetica della popolazione dializzata nel Polo Ospedaliero della AsI Roma 4. Interventi formativi multispecialistici mirati assicureranno ai pazienti di ricevere e rinforzare conoscenze per raggiungere: obiettivi in termini di qualità di vita, miglioramento del self-management, prevenzione di accessi impropri in Pronto Soccorso e scelta del proprio stile di vita.

\section{ID-13}

STUDIO OSSERVAZIONALE SULL'EFFICACIA DEL DISPOSITIVO DI MEDICAZIONE AVANZATA IN DACC (GARZA DI ACETATO VERDE) NEGLI ACCESSI VASCOLARI IN EMODIALISI (BUTTONHOLE E CVC) L. Sbarbati, F. Dottori, D. Lucidi, A. Fraboni, R. Cittadini, F. Esposito, S. Picciaiola, LaL.ura Galeassi, K. Monteverdi, M. Zeiler U.O. Nefrologia e Dialisi, Ospedale "Carlo Urbani", Jesi (AN)

Introduzione: La prevenzione delle infezioni dell'accesso vascolare è di fondamentale importanza per la sua durata e la gestione, sia esso un CVC o una FAV. Le medicazioni avanzate in DACC, garza in tessuto di acetato di colore verde impregnata di un derivato degli acidi grassi, inducono con la loro azione idrofobica i microorganismi a legarsi velocemente alla fibra della medicazione, quindi potrebbero prevenire infezioni e coadiuvarne la guarigione in caso di exit-site "dubbi".

Materiali e Metodi: Abbiamo condotto uno studio osservazionale su 16 pazienti per 18 sedute dialitiche: 5 pazienti con Buttonhole, 3 pazienti con CVCt e 9 pazienti con CVCp. Abbiamo applicato la medicazione seguendo i protocolli e seguito la scala dello score dell'E.S. secondo i criteri di Twardowski per segnalare lo stato dell'E.S. e del Buttonhole. I 3 pazienti con CVCt mostravano segni rossore, di cui 1 con secrezione sieroematica score 2 (tampone neg.). Dei 9 pazienti con CVCp, 3 presentavano segni di rossore score 1 (tampone neg. emocoltura neg.) Dei 5 pz con Buttonhole, 2 avevano precedenti episodi d'infezione del sito di venipuntura.

Risultati: CVCt: sin dalla seconda medicazione, si evidenziano una progressiva riduzione della deiscenza della ferita, la scomparsa del dolore e la diminuzione dell'essudato con diminuzione del dolore. L'essudato sieroso si è risolto dalla terza medicazione. Dopo la sesta medicazione, l'E.S. si presenta buono, senza segni di infiammazione e secrezione con punteggio 0 dell'E.S. Scoring System. CVCp: dalla terza medicazione, si evidenzia un miglioramento del rossore, ma permane una crosticina secca. Dalla settima medicazione, E.S. perfetto. BH: dalla terza medicazione le crosticine appaiono più piccole e piatte.

Conclusioni: L'uso del dispositivo in DACC ha evidenziato notevoli miglioramenti nella gestione degli arrossamenti e della secrezione dell'E.S. (score $1-2)$; il dispositivo è di facile applicazione e, se l'E.S. ha score 0 , può restare in sito fino a 4-7 giorni con medicazione trasparente. II suo uso per la FAV per i pazienti con tecnica di venipuntura Buttonhole è efficace al $60 \%$ dei casi nel ridurre le crosticine, ma vincente per il gradimento dei pazienti, che si sentono protetti dal presidio che tengono fino alla seduta successiva; d'altro canto, alcuni pazienti hanno sviluppato un'allergia al cerotto trasparente in dotazione e, quindi, abbiamo dovuto sospenderne l'uso fino all'arrivo del presidio dotato di cerotto al silicone. Risulta ben tollerato dalla maggioranza dei pazienti.

\section{ID-04}

IL CATETERE VENOSO CENTRALE IN EMODIALISI: STUDIO SULL'EFFICACIA DELLA CLOREXIDINA VERSUS L'IPOCLORITO DI SODIO

I. Parisi $^{1}$, V. Carlucci ${ }^{2}$, F. Valerio ${ }^{3}$, G. Petruzzelli ${ }^{3}$, M. Maffei ${ }^{3}$, A. Maggiulli $^{3}$, M.L. Biga ${ }^{3}$, M. Giannetto ${ }^{3}$, S. Di Paolo ${ }^{3}$

${ }^{1}$ Infermiere presso U.O.C. Anestesia e Rianimazione P.O. Barletta, Barletta (BT) ${ }^{2}$ Infermiere di Direzione Medica, Presidio Andria-Canosa, Andria (BT)

${ }^{3}$ U.O.C di Nefrologia e Dialisi - P.O. "Mons. Dimiccoli”, Barletta (BT)

Introduzione: Le infezioni nosocomiali rappresentano le complicanze più frequenti tra i soggetti che, nella loro quotidianità, si confrontano con la realtà ospedaliera, come nel caso del paziente emodializzato attraverso CVC (catetere venoso centrale), che rappresenta il soggetto di osservazione del seguente studio.

Lo studio nasce con la finalità di valutare se, attraverso una gestione differente per tipologia di disinfezione, sia possibile ridurre l'incidenza delle infezioni dell'exit-site del CVC. Gli obiettivi, dunque, sono stati: definire se l'antisepsi cutanea effettuata con clorexidina al $2 \%$ al cambio di medicazione (disinfettante di base alcolica) favorisca la riduzione delle infezioni cutanee dell'exit-site rispetto all'utilizzo di ipoclorito di sodio allo 0,05\%; valutare se il tipo e la sede di inserzione del CVC, i giorni di permanenza del CVC, la fascia d'età del paziente, il sesso e il BMI possono modificare l'incidenza di infezione in corrispondenza dell'exit-site.

Materiali e Metodi: I dati sono stati raccolti e le osservazioni sono state effettuate su un campione di $\mathrm{n}^{\circ} 98$ pazienti valutando le disinfezioni dell'exit-site del CVC opportunamente registrate. Lo studio, del quale la parte osservazionale ha avuto una durata di 12 mesi (anno 2018), è stato condotto nel Centro Dialisi dell'UOC di Nefrologia e Dialisi dell'Ospedale "Mons. R. Dimiccoli” di Barletta. In merito alla parte retrospettiva dello studio (anno 2017), indispensabile per il confronto tra i due tipi di disinfezione (ossia quella ancora attualmente utilizzata, effettuata con clorexidina, e quella eseguita in passato con ipoclorito di sodio), i dati sono stati rilevati consultando le schede infermieristiche archiviate dei pazienti considerati nel campione (pazienti trattati con ipoclorito di sodio per la disinfezione dell'exit-site), mentre, per la parte di studio osservazionale, i dati sono stati raccolti attraverso osservazione e per mezzo di una scheda di valutazione, comprendente la scala Visual Exit-site Score (acronimo VES) CVC per la rilevazione dei segni di flogosi (pazienti che hanno effettuato la disinfezione con la clorexidina). I dati sono stati riportati in file excel, per l'elaborazione mediante il software statistico SPSS 19 demo. Per la statistica descrittiva e inferenziale è stato utilizzato il T-test per campioni indipendenti, fissando un intervallo di confidenza (IC) al 95\%.

Risultati: I punteggi medi ottenuti per mezzo della scala di valutazione VES CVC si sono mostrati a favore di una cute integra nel caso di utilizzo di clorexidina come antisettico cutaneo, rispetto ai segni di flogosi predominanti nella situazione in cui l'antisepsi è stata effettuata con disinfettante a base di ipoclorito di sodio allo $0,05 \%$.

I risultati conseguiti hanno dimostrato l'efficacia della disinfezione dell'exitsite effettuata con la clorexidina al $2 \%$ nella riduzione dell'incidenza di infezione rispetto a quelle eseguite con ipoclorito di sodio allo 0,05\%, $p<0,05$. $\mathrm{Si}$ è riscontrato che l' $89 \%$ dei pazienti che hanno effettuato la disinfezione con ipoclorito di sodio ha manifestato un segno di infezione, a differenza del $46 \%$ dei pazienti sottoposti a disinfezione con clorexidina. Nel complesso, si è riscontrata una riduzione della presentazione dei segni di infezione del 66\% dal momento in cui è stata adottata la clorexidina per la disinfezione dell'exitsite del CVC.

La valutazione delle variabili tipo di CVC, sede di inserzione, giorni di permanenza, nonché fascia d'età del paziente, sesso e BMI, non ha dimostrato l'incidenza di queste ultime nella riduzione delle infezioni, $p>0,05$.

Conclusioni: L'esito dello studio si è definito a favore della clorexidina come antisettico cutaneo efficace nella riduzione delle infezioni CVC correlate. Nello specifico, è stato l'utilizzo di una soluzione di base alcolica, come la clorexidina $2 \%$ in questione, che ha portato delle notevoli differenze rispetto all'utilizzo di ipoclorito di sodio allo $0,05 \%$ nell'esecuzione della disinfezione a livello dell'exit-site. L'indicazione verso un tipo di disinfezione che comporta l'adozione di tale soluzione antisettica (clorexidina) è utile per prevenire e gestire le infezioni cutanee in corrispondenza dell'exit-site, in modo tale da evitare che queste possano diventare sistemiche (CRBSI, infezione del flusso di sangue relativo al catetere) e compromettere o aggravare la salute stessa del paziente. 


\section{ID-20}

AGHI CANNULA PER EMODIALISI VS AGHI IN METALLO, LA NOSTRA ESPERIENZA

S. Cappelletti, D.C Massarenti

Unità Semplice di Nefrologia e Emodialisi, Asst Lariana, S. Fermo della Battaglia, Como (CO)

Introduzione: Ruolo fondamentale per la somministrazione di un'adeguata dose dialitica e determinante per il successo o il fallimento della terapia dialitica stessa lo riveste l'accesso vascolare. La FAV nativa (FAVn) o la FAV protesica (FAVp) risultano essere un punto critico nell'assistenza infermieristica al paziente dializzato. La loro disfunzione è la maggiore causa di morbilità e di ospedalizzazione. La stenosi e la trombosi sono le complicanze principali della fistola nativa e protesica, che aumentano la crescente dipendenza dai cateteri venosi centrali, incrementando il rischio di infezioni, mortalità e costi sanitari. Le Linee Guida portano l'accesso vascolare al centro delle cure, raccomandando un regolare monitoraggio del flusso e considerando gli episodi trombotici come indicatori della qualità del trattamento dialitico stesso. Molti pazienti temono l'emodialisi perché comporta l'inserimento di aghi, in più occasioni in una fistola artero-venosa (FAV). Le preoccupazioni per $\mathrm{i}$ pazienti sono sempre state il dolore, il successo della cannulazione e il completamento del trattamento dialitico. La venipuntura può essere effettuata o con ago in metallo a punta tagliente a becco di flauto o utilizzando aghi cannule per emodialisi. Abbiamo voluto fare uno studio osservazionale per valutare eventuali differenze tra le due tipologie di dispositivo, concentrandoci su pazienti portatori di FAV protesiche, per la scarsa bibliografia presente in merito.

Materiali e Metodi: 14 pazienti portatori di graft, sottoposti a emodialisi trisettimanale, sono stati monitorati per 6 mesi, cambiando dall'uso precedente di aghi in metallo (16 G) all'uso di quelli con ago cannula (16 G). Nel corso dell'osservazione sono stati monitorati i seguenti parametri: valori medi di pressione arteriosa (PA) e pressione venosa (PV), tempo di emostasi, compliance del paziente (scala di misurazione del dolore NRS) ed eventuali complicanze.

Risultati: Il confronto dei dati con i tre mesi in cui i pazienti portatori di protesi erano sottoposti alla puntura con aghi in metallo e dei dati nei tre mesi successivi, in cui gli stessi pazienti, portatori di protesi, erano sottoposti alla puntura di aghi cannula fistola ha portato a una tendenza positiva per gli aghi cannula in termini medi di compliance del paziente (misurazione del dolore con scala NRS) e tempi di emostasi. I valori pressori, al contrario, sembrerebbero mostrare una tendenza positiva, invece, per gli aghi in metallo.

Conclusioni: Anche se i risultati di questo studio sono limitati alla nostra esperienza su un campione ridotto di pazienti e dovrebbero essere confermati da analisi più robuste, sembrano protendere, in ambito di emodialisi, per l'utilizzo di aghi cannula per emodialisi in quei pazienti con elevati tempi di emostasi e dolore alla venipuntura. I valori pressori sembrerebbero mostrare una tendenza positiva per gli aghi in metallo (PA: $-166 \mathrm{mmHg}$ vs $-227 \mathrm{mmHg}$ e PV: $207 \mathrm{mmHg}$ vs $225 \mathrm{mmHg}$ ). Per concludere, possiamo affermare che la nostra esperienza, da un lato, riconosce i vantaggi tecnici di questi dispositivi rispetto agli aghi metallici e, dall'altro, vede nel lavoro di equipe multidisciplinare, in cui le competenze dei professionisti sono al servizio del paziente, la strategia ottimale per la gestione degli accessi vascolari.

\section{ID-21}

LA MALATTIA RENALE CRONICA: QUALITÀ DI VITA, ANSIA E DEPRESSIONE IN UN GRUPPO DI PAZIENTI IN FASE PRE-DIALITICA G. Gerbino ${ }^{1}$, G. Soragna ${ }^{1}$, D. Curci ${ }^{2}$, D. Fazzari ${ }^{2}$, M. Bauducco ${ }^{1}$, A. Panunzi ${ }^{1}$, L. Fabbrini ${ }^{1}$, P. Chiazzolla ${ }^{1}$, C. Vitale ${ }^{1}$

${ }^{1} \mathrm{SC}$ Nefrologia e Dialisi, AO Ordine Mauriziano, Torino (TO)

${ }^{2} \mathrm{Cdl}$ in Infermieristica, Università degli Studi di Torino, Torino (TO)

Introduzione: Numerosi studi evidenziano, nei pazienti affetti da patologie croniche, un peggioramento della qualità di vita, un'alta incidenza di disturbi dell'umore e un aumentato rischio di morbilità e mortalità.

In campo nefrologico, la letteratura è prevalentemente dedicata a studi condotti su soggetti in trattamento dialitico; questo lavoro si prefigge, invece, di valutare l'incidenza di problemi psicologici in pazienti affetti da insufficienza renale cronica (IRC) in trattamento conservativo non in dialisi.

Materiali e Metodi: Sono stati studiati 155 pazienti (M/F: 104/51), seguiti presso l'ambulatorio della Malattia Renale Avanzata (Stadi IV e V), dei quali il $51 \%$ di età > 80 anni, il $40,6 \%$ di età $65-79$ anni e l'8,4\% di età $30-64$ anni, attraverso la somministrazione di due questionari: Hospital Anxiety and Depression Scale (HADS) per la valutazione dell'ansia e della depressione e
SF-12 versione italiana per l'analisi della salute fisica (PCS) e mentale (MCS). Il questionario HADS restituisce uno score diagnostico; come riferimento dei punteggi SF-12, sono state considerate le rilevazioni ISTAT sulla popolazione generale.

Risultati: I risultati emersi dall'HADS evidenziano che 29 pazienti $(18,7 \%)$ mostrano ansia clinicamente rilevante e $22(14,2 \%)$ evidenziano situazioni potenzialmente evolutive in senso psicopatologico. Per quanto riguarda la depressione, 37 pazienti $(23,9 \%)$ hanno mostrato segni di patologia, mentre altri $37(23,9 \%)$ hanno mostrato condizioni borderline.

L'analisi del questionario SF-12 ha indicato per la PCS una mediana di 35 (dato ISTAT: 54) e per la MCS una mediana di 49 (dato ISTAT: 52).

Conclusioni: La ricerca condotta ha evidenziato che i pazienti con patologia renale cronica in fase pre-dialitica mostrano misure di qualità della vita, dell'ansia e della depressione clinicamente rilevanti. I risultati sono compatibili con l'ipotesi che suggerisce di considerare questo gruppo di persone come distinte dalla popolazione generale, bisognose di una presa in carico medica e psicologica specifica a loro dedicata. In particolare, si può argomentare a favore di una figura professionale di riferimento che abbia formazione di base da psicologo e auspicare, così, una crescente specializzazione degli interventi psicologici e medici che possano offrire ai pazienti quella presa in carico come persone nella loro interezza.

\section{STRESS E BURNOUT}

\section{ID-14}

\section{EFFETTO FILTRO: IL BURNOUT IN DIALISI. INDAGINE CONOSCITIVA} E STRUMENTI DI INTERVENTO

F. Manari, S. Mennilli, L. Di Liberato, M. Bonomini

Unità Operativa di Nefrologia e Dialisi, Ospedale Clinicizzato SS. Annunziata, Chieti (CH)

Introduzione: Nell'ambito di un progetto di ricerca condotto presso l'Unità di Nefrologia e Dialisi dell'Ospedale SS. Annunziata di Chieti nell'anno 2019, I'inserimento della figura dello psicologo ha permesso la realizzazione di un programma di valutazione del burnout nel personale medico e infermieristico. Costretto a confrontarsi costantemente con alti livelli di sofferenza e con vissuti di perdita legati alle funzioni organiche, alle limitazioni imposte dalla malattia, alla propria autonomia e alle aspettative future e con vissuti legati alla morte, il personale sanitario è inevitabilmente esposto al rischio di burnout.

Materiali e Metodi: L'obiettivo dell'intervento psicologico è quello di preservare l'elemento che garantisce il progetto di cura per il paziente, ossia la relazione di aiuto. Attività di formazione e colloqui di sostegno al disagio del personale contribuiscono a evitare l'acuirsi di tensioni o di contrasti tra gli operatori, tutelando, così, la qualità del contesto di cura del malato.

Lo strumento utilizzato per rilevare i livelli di burnout è il Maslach Burnout Inventory. Il campione di riferimento è composto da 21 soggetti.

Risultati: Ciò che è emerso confrontando i punteggi delle tre dimensioni (Esaurimento Emotivo, Depersonalizzazione, Realizzazione Personale) nei livelli di burnout basso, medio e alto, prima dell'intervento psicologico e dopo, è una leggera riduzione dei punteggi alti e un piccolo miglioramento dei punteggi bassi, con aumento dei punteggi medi di tutte e tre le dimensioni, in particolare nell'area della Realizzazione Personale.

Conclusioni: L'intervento psicologico rivolto al personale sanitario risulta, quindi, efficace sia come strumento di prevenzione del burnout, sia come mezzo utile nell'individuazione di casi di operatori in difficoltà, sia come risorsa nell'accrescere la motivazione nello svolgimento della propria professione. Ciò consente di lavorare sulle modalità comunicative e sugli stili difensivi utilizzati nella relazione con il paziente, al fine di esplorare le percezioni emotive di sé e del paziente che possono condizionare la relazione terapeutica.

\section{ID-26}

\section{STRESS E BURNOUT, FENOMENI PRESENTI NEGLI INFERMIERI IN} DIALISI?

A. Pizzo ${ }^{1}, \underline{\text { S. Porzio }}^{1}$, G. Di Pietro ${ }^{2}$, D. Dileo ${ }^{2}$, V. Neiviller ${ }^{1}$, M.T. Parisotto ${ }^{3}$

${ }^{1}$ NephroCare Italia, Coordinamento Infermieristico, Napoli (NA)

${ }^{2}$ Freelancer, Taranto (TA)

${ }^{3}$ Freelancer, Milano (MI)

Introduzione: Il termine stress è inteso come una risposta aspecifica dell'organismo a uno stimolo esterno. 
Stress e burnout sono spesso collegati tra loro nel personale infermieristico. Il burnout viene, infatti, definito come una risposta psicologica allo stress cronico lavorativo di natura interpersonale ed emozionale che appare nei professionisti che lavorano direttamente con i pazienti.

Lo scopo di questo lavoro è stato quello di valutare il livello di stress e di eventuale presenza di burnout all'interno dei team di lavoro infermieristici di due Centri Dialisi.

Materiali e Metodi: Nell'ottobre del 2018 è stato somministrato il Burnout Questionnaire (BQ) standard dell'American Public Welfare Association, composto da 25 domande mirate alla valutazione del grado di stress del soggetto e della sua possibile evoluzione in burnout. I risultati hanno fornito cinque diverse categorie relative allo stress, divise in stress assente, lieve, moderato, alto e molto alto con presenza di burnout.

Nel novembre del 2018 è stato chiesto al personale infermieristico coinvolto nello studio, di compilare di un questionario anonimo e in modalità on-line, per identificare i fattori di maggiore stress. Da gennaio 2019, è stato attivato dall'ufficio human resources un progetto aziendale nazionale di supporto ai professionisti sanitari attraverso degli incontri con un team di psicologi, utile al miglioramento delle relazioni, della comunicazione e dei fattori di stress rilevati.

Risultati: Il campione è composto da 19 infermieri, di cui il 68,4\% donne.

La somministrazione del BQ evidenzia che il 5,26\% non presenta nessun grado di stress, il $36,85 \%$ uno stress lieve, il $42,10 \%$ uno stress moderato, il $15,79 \%$ alti livelli di stress e il possibile inizio di burnout e lo $0 \%$ i massimi livelli di stress con burnout conclamato.

Tra i fattori di stress riscontrati si evincono: comportamenti scortesi de pazienti per il $42,1 \%$, la routine lavorativa per il $42,1 \%$, conflitti interpersonali per il 31,6\%, la mancanza di collaborazione tra colleghi per il 31,6\%, problemi relativi alle relazioni personali e/o alla comunicazione nel team infermieristico per il $26,3 \%$.

Conclusioni: Il presente lavoro mostra che lo stress e l'esposizione al rischio di burnout, così come illustrato nelle fonti bibliografiche consultate, possono essere fenomeni presenti negli infermieri che lavorano in dialisi, poiché quotidianamente a contatto con le difficoltà e le sofferenze dei propri assistiti, affetti da patologia renale cronica. L'identificazione della presenza di stress e l'analisi dei fattori che lo determinano sono strategie importanti per poter pianificare degli interventi correttivi utili a ridurre le probabilità di giungere al burnout, impedendo che questo interferisca con gli aspetti personali e organizzativi e con le capacità professionali degli infermieri di soddisfare i bisogni assistenziali dei pazienti.

\section{APPROCCIO OLISTICO}

ID-27

\section{APPROCCIO OLISTICO AI PAZIENTI IN EMODIALIS}

A. Kielbasa ${ }^{1}$, N. Palermo ${ }^{1}$, A. Indelicato ${ }^{1}$, F. Savarese ${ }^{1}$, S. Caldiero ${ }^{1}$, R. D'Amato ${ }^{1}$ R. Abborrito ${ }^{1}$ M. Migliore ${ }^{2}$, A. Pizzo ${ }^{3}$

${ }^{1}$ NephroCare Italia, Centro Dialisi NephroCare ex Enne-E, Napoli (NA)

${ }^{2}$ Freelancer, Napoli (NA)

${ }^{3} \mathrm{NephroCare}$ Italia, Coordinamento Infermieristico, Napoli (NA)

Introduzione: Siamo nell'epoca della medicina di precisione, della medicina ultra-specialistica, che ha certamente condotto a risultati e progressi di rilievo. Tuttavia, si è perso il concetto di medicina olistica, che adottava come suo credo "curare l'anima per curare il corpo". Parallelamente, il nursing, che, per sua natura intrinseca, è olistico, a causa di diverse condizioni organizzative e culturali, ha favorito una dimensione infermieristica con una visione dell'uomo meno globale e con maggiore focus al corpo malato, che ha bisogno di essere assistito.

La salute non è la sola assenza di malattia o infermità, ma uno stato completo di benessere fisico, mentale, spirituale e sociale. Nel nostro centro di emodialisi, attraverso il coinvolgimento di tutto lo staff multidisciplinare, da qualche anno abbiamo, quindi, sposato questo concetto, che si perde, in realtà, nella notte dei tempi, giacché già Platone affermava: "Non muovere mai l'anima senza il corpo, né il corpo senza l'anima, affinché difendendosi l'uno con l'altra, queste due parti mantengano il loro equilibrio e la loro salute".

Materiali e Metodi: In questi ultimi anni, abbiamo portato avanti 2 progetti ispirati a questi concetti, coinvolgendo tutti i pazienti:
1) "Io non sono la malattia, ma sono tutti i colori del mondo" Progetto di Make-up, svoltosi nell'anno 2017. Al progetto hanno partecipato 6 pazienti tra i 40 e i 70 anni e sono stati coinvolti una truccatrice e un fotografo professionisti.

2) "Io non sono la dialisi, io faccio la dialisi. Passerella in poesia e musica", svoltosi nell'anno 2018 e al quale hanno partecipato 10 pazienti di entrambi i sessi tra i 30 e gli 80 anni, insieme alla quasi totalità del personale sanitario.

Risultati: Alla fine del primo progetto è stato distribuito ai partecipanti un questionario di gradimento, dal quale si evinceva che tale attività è risultata utile e di supporto sia ai pazienti che ai caregiver. Come risultato finale del secondo progetto, tutti insieme abbiamo scelto, con l'autorizzazione dell'autore, liriche dal testo "I'arte di essere fragili" di Alessandro d'Avenia e diversi brani musicali, ognuno dei quali rispecchiava l'indole e il vissuto dei partecipanti. Nella serata finale, ogni partecipante ha sfilato, mentre l'oratore declamava le liriche accompagnato da una base musicale.

Conclusioni: Questa tipologia di progetti aiuta i pazienti ad affrontare meglio la malattia, incoraggia e migliora l'approccio olistico del personale sanitario nel garantire la dovuta attenzione alla qualità della vita e allo stato emozionale ed emotivo dei pazienti e sostiene i caregiver, dando a tutti la consapevolezza che le strategie terapeutiche non si avvalgono solo di farmaci e di tecnologia, ma anche del potere delle parole e delle idee.

\section{NUOVE STRATEGIE DI NURSING}

\section{ID-07}

NUOVE STRATEGIE DI NURSING PER UNA MIGLIORE DOSE DIALITICA

G. Petruzzelli, M. Biga, A. Cisternino, A. De Vivo, R, Falanga, R. Lemma, M. Maffei, A. Maggiulli, L. Pinto, R. Pischetola, A. Rizzi, M. Scardigno, F. Valerio, P. Magarelli, M. Giannetto, S. Di Paolo U.O. Nefrologia-Dialisi Ospedale "Mons. R. Dimiccoli”, Barletta - Nephrocare, Trani (BT)

Introduzione: L'emodialisi quale terapia sostitutiva della funzione renale è una tecnica ormai ben definita. Gli studi eseguiti in questi ultimi 35-40 anni e la ricerca tecnologica hanno, infatti, permesso di ottimizzare e personalizzare le terapie ottenendo risultati prima insperati, soprattutto in materia di qualità di vita offerta al paziente. La giusta dose emodialitica è un target cruciale, in quanto determina la sopravvivenza e il benessere del paziente emodializzato. Numerosi studi hanno dimostrato una correlazione tra la dose di dialisi somministrata e la morbilità e la mortalità del paziente. L'adeguatezza dialitica è convenzionalmente valutata misurando la clearance frazionata dell'urea (KT/V), che, per ogni singolo trattamento, dovrebbe essere superiore o almeno uguale a 1,2. È stato dimostrato che, ad ogni incremento di 0,1 del KT/V, corrisponde una diminuzione del $7 \%$ della probabilità di morte.

Materiali e Metodi: II nostro studio-progetto ha voluto valorizzare la misura in continuo online del diagramma della clearance dell'urea con i device Diascan od OCM con la disponibilità di alcuni monitor di dialisi, dando rilievo al ruolo dell'eparina nel modulare i valori di KT/V in relazione ai dosaggi utilizzati. L'heparin study ha richiesto un periodo osservazionale ed elaborativo dei dati di circa due mesi, ottobre-novembre 2019, e ha coinvolto tutti i pazienti del centro dialisi dell'Ospedale "Mons. R. Dimiccoli" di Barletta. I parametri presi in considerazione nello studio sono stati: assetto coagulativo, assetto lipidico, terapia antiaggregante e TAO domiciliare, monitoraggio della clearance $(\mathrm{K})$ a inizio e fine dialisi, monitoraggio del KT/V teorico ed effettivo, valori di attacco di $\mathrm{Hb}$ e di Hct, tipologia di trattamento dialitico e tipo di anticoagulazione del circuito extracorporeo, se eparina sodica o eparina a basso peso molecolare.

Risultati: Lo studio ha messo in rilievo come il dosaggio dell'anticoagulante sia fondamentale nell'influenzare il KT/V: variazioni incrementali dell'anticoagulante hanno conseguito un miglioramento dei livelli di clearance nel 73,7\% dei pazienti, mentre il $26,3 \%$ non ha mostrato alcuna variazione (non responder). La dose di anticoagulante somministrata a ciascun paziente è stata calcolata in accordo con le indicazioni delle "European Best Practice Guidelines for Hemodialysis".

Conclusioni: Lo studio ha consentito di: 1) apprezzare l'utilità dei diagrammi di clearance forniti dai device a disposizione, in quanto capaci di fornire informazioni in tempo reale sull'efficienza dialitica del singolo paziente; 2) 
eliminare l'utilizzo di dosi standard di anticoagulante intradialitico e di somministrare dosi personalizzate, innalzando il beneficio del trattamento in termini depurativi, senza eventi avversi.

\section{CASE REPORT}

\section{ID-06}

MONITORAGGIO DELLA PRESSIONE PRE-FILTRO IN DIALISI COME INDICATORE DI TROMBOSI VENOSA PROFONDA

G. Petruzzelli ${ }^{1}$, M. Maffei $^{1}$, A. Maggiulli ${ }^{1}$, A, Cisternino ${ }^{1}$, R. Falanga ${ }^{1}$,

L. Pinto ${ }^{1}$, I. Parisi ${ }^{2}$, N. Lacerenza ${ }^{2}$, F. Valerio ${ }^{1}$, M.L. Biga ${ }^{1}$, R. Pischetola ${ }^{1}$,

M. Giannetto ${ }^{1}$, S. Di Paolo

${ }^{1}$ UOC Nefrologia-Dialisi Ospedale "Mons. R. Dimiccoli", Barletta (BT)

${ }^{2}$ Laureati in infermieristica, Barletta (BT)

Introduzione: I manuali di istruzioni operative dei monitor ci forniscono informazioni sulla pressione pre-filtro e, nello specifico, su:

1) efficienza funzionale e depurativa dei capillari del filtro;

2) modulazione automatica dei volumi di infusione nelle metodiche convettive. Nell'ultimo biennio, l'attento monitoraggio della pressione pre-filtro ci ha permesso di individuare 2 casi clinici strettamente affini tra loro, dove l'aumento della pressione pre-filtro non era attribuibile a un esaurimento dei capillari del filtro, ma, bensì, a una trombosi venosa profonda della vena ospitante il CVC femorale.

Materiali e Metodi: Presentiamo il caso clinico del Sig. C.R., paziente di 85 anni, diabetico, iperteso, cardiopatico e portatore di pace-maker, in trattamento TAO, al quale viene confezionata una fistola artero-venosa distale sinistra, con ingresso in dialisi nell'ottobre del 2017. Successivamente, per sindrome del braccio grosso, si procede alla chiusura della FAV, con l'impianto di un CVC long term per via femorale destra per esaurimento del patrimonio vascolare. Si premette che il paziente non ha mai eseguito una procedura dialitica in modalità monoago. II team infermieristico ha osservato che nella prima ora di dialisi si verificava un incremento progressivo della pressione pre-filtro, mentre la Pressione Venosa e la Pressione Arteriosa rimanevano pressoché invariate, con riduzione della pressione pre-filtro alla sospensione della Ultra Filtrazione.

Risultati: Per differenziare l'impaccamento del filtro dall'emoconcentrazione, si è proceduto a incannulare una vena dell'avambraccio sinistro che ospitava la FAV e si è utilizzato l'accesso vascolare come scarico per ridurre il ricircolo e, di conseguenza, l'emoconcentrazione, con esito positivo e riduzione della pressione pre-filtro. Aggiornato il medico sulle dinamiche intradialitiche relative all'incremento della pressione pre-filtro, viene disposta una valutazione radiologica del paziente con Angio-TC. L'esame, eseguito a luglio del 2019, descrive una trombosi endoluminale in corrispondenza del tratto intraepatico della vena cava inferiore e una presenza di trombi in corrispondenza della vena femorale comune destra.

Conclusioni: Questa esperienza, supportata da un'attenta osservazione della pressione pre-filtro, ci ha permesso di individuare un probabile problema di natura trombotica dell'accesso vascolare, ponendo in seconda ipotesi l'impaccamento dei capillari del filtro, in quanto, a un rallentamento del flusso ematico del vaso ospitante, corrisponde un aumento dell'emoconcentrazione. È stato possibile eseguire il monitoraggio solo su apparecchiature che hanno in dotazione il device della pressione pre-filtro. misurato mediante sensore dedicato e visibile sul display per tutta la durata del trattamento. L'obiettivo futuro è confermare attraverso l'osservazione di più casi clinici l'utilità di questo trasduttore spostando l'attenzione dell'operatore anche sulle FAV native.

\section{ADERENZA TERAPEUTICA}

\section{ID-17}

VALUTAZIONE DELLO STATO NUTRIZIONALE NEI PAZIENTI DEL CENTRO DIALISI DI MATERA

\section{G. Zaccaro}

Dialisi, Presidio Ospedaliero Matera (MT)

Introduzione: Chi lavora in dialisi si trova spesso ad affrontare il problema della malnutrizione. Un'attenta valutazione dello stato nutrizionale è la premessa per intervenire precocemente e migliorare la prognosi e la qualità di vita di questi pazienti.

Per lo screening nutrizionale ci sono vari test, questionari e punteggi, che prendono in considerazione: I'anamnesi del soggetto, l'apporto di nutrienti calorico e proteico, l'antropometria e la composizione corporea e test di laboratorio. Quello proposto da Linee Guida sull'alimentazione in modo specifico per i pazienti in emodialisi è il questionario MIS Malnutrition Inflammation Score.

Materiali e Metodi: Nel centro dialisi di Matera ci sono 71 pazienti e, di questi, 58 sono stati arruolati nello studio. È stata eseguita la bioimpedenziometria a tutti i pazienti e solo 4 hanno un angolo di fase inferiore o uguale a 4 (valore importante come indice di mortalità).

Dalla biochimica clinica, abbiamo visto che la maggior parte dei pazienti presenta un valore di albumina inferiore a $3,8 \mathrm{~g} / \mathrm{dL}$, ma superiore a $3 \mathrm{~g} / \mathrm{dL}$, indicativo di una lieve malnutrizione.

Ai pazienti è stato consegnato il diario alimentare, nel quale hanno registrato i consumi alimentari per una settimana. Si è visto che i valori di intake proteici e calorici sono molto bassi rispetto al limite inferiore.

Mettendo in relazione il valore medio dell'intake proteico con il valore medio del Npcr (calcolo che fornisce dati oggettivi di intake proteici), abbiamo riscontrato una grande differenza tra i due valori. Un grafico di dispersione ci ha confermato come più basso è l'intake calorico e più aumenta la differenza tra intake proteico e Npcr.

Questo è riconducibile sia al limite del diario alimentare nella corretta compilazione sia all'ipercatabolismo, in cui parte del valore del Npcr deriva dal catabolismo delle proteine muscolari. Sono, quindi, pazienti che hanno bisogno di un supporto nutrizionale che serva sia a limitare l'ulteriore perdita proteico-energetica sia a ricostruire le riserve ridotte.

Risultati: Secondo il questionario MIS, il 56\% dei pazienti presenta una malnutrizione lieve e il $18 \%$ una malnutrizione grave.

I dati evidenziano che un'ampia percentuale di pazienti presenta una massa cellulare ridotta e un apporto proteico inferiore ai valori raccomandati.

Conclusioni: Negli ultimi anni, la gestione infermieristica della nutrizione è diventata sempre più rilevante.

L'infermiere di nefrologia e dialisi ha il compito di riuscire a prevenire, in collaborazione con l'equipe medica e il servizio di dietologia, la malnutrizione o un'errata alimentazione, responsabile del rischio di morbilità e di mortalità.

\section{ID-25}

\section{ADERENZA TERAPEUTICA DEI PAZIENTI IN EMODIALIS}

V. Neiviller ${ }^{1}$, M. Cerreto ${ }^{2}$, A. Pizzo ${ }^{1}$, M.T. Parisotto ${ }^{3}$

${ }^{1}$ NephroCare Italia, Coordinamento Infermieristico, Napoli (NA)

${ }^{2}$ NephroCare Italia, Centro Dialisi NephroCare Vomero, Napoli (NA)

${ }^{3}$ Freelancer, Milano (MI)

Introduzione: L'aderenza terapeutica è determinante per il successo di tutti i trattamenti terapeutici. La mancata aderenza, "non-compliance", di un paziente alle prescrizioni farmacologiche è, oggi, universalmente riconosciuta come un problema frequente che aumenta i costi dell'assistenza. Nei pazienti in HD, una "non adesione" alla terapia può avere importanti ricadute, sia di tipo clinico che di tipo economico, con un aumento del numero e della durata delle ospedalizzazioni.

Questo lavoro intende valutare la compliance terapeutica dei pazienti in emodialisi e si interroga sul ruolo dell'infermiere nel miglioramento degli outcome clinici.

Materiali e Metodi: A novembre 2018 è stato somministrato, su un campione di 108 pazienti di due centri dialisi, la Morisky Medication Adherence Scale (MMAS-8), composta da 8 semplici domande con possibilità di risposta: Sì (0) e NO (1). II punteggio totale ottenuto ha permesso di suddividere il campione in: alta (4 punti), media (2-3 punti) e bassa (0-1 punti) aderenza terapeutica. La compliance dialitica è stata misurata analizzando i trattamenti dialitici degli ultimi 3 mesi attraverso i valori medi di KT/V, tempo di dialisi effettivo e sedute dialitiche disertate e la compliance dietetica con i valori di laboratorio per Fosforo e Potassio PreHd dello stesso periodo; per il corretto introito di liquidi, è stato valutato lo stato di idratazione attraverso esame bioimpedenziometrico multifrequenza.

Risultati: II questionario è stato somministrato in 2 Centri Dialisi su un campione di 108 pazienti: 102 pazienti (94\%) hanno risposto al sondaggio, il $66,7 \%$ rappresentato da donne, età media 69,1 anni. II $68,6 \%$ dei 
partecipanti è risultato avere un'alta aderenza alla terapia farmacologica prescritta, il $20,6 \%$ media e il $10,8 \%$ bassa. L' $87,96 \%$ dei pazienti ha raggiunto un $\mathrm{KT} / \mathrm{V}$ medio $>1,4$ e il $5,6 \%$ aveva un $\mathrm{KT} / \mathrm{V}<1,2$, il tempo di dialisi medio è stato di 235,62 minuti e lo $0,37 \%$ dei trattamenti risultava disertato. II $17,6 \%$ mostrava una fosforemia media $>5,5 \mathrm{mg} / \mathrm{dL}$, il $22,2 \%$ riscontrava una potassiemia media $>5,5 \mathrm{mEq} / \mathrm{L}$ e il $29,6 \%$ risultava avere un'elevata sovraidratazione PreHd.
Conclusioni: Migliorare l'aderenza alle terapie è una priorità globale per garantire sicurezza delle cure, qualità della vita e sostenibilità delle cure. In questo studio, si evidenzia che gli infermieri di dialisi possono rivestire un ruolo fondamentale nell'empowerment del paziente in emodialisi, al fine di renderlo protagonista del suo percorso di cura, e nel valutare e nel promuovere I'aderenza terapeutica dialitica, farmacologica e dietetica, attraverso interventi infermieristici rivolti a lui, alla sua famiglia e ai caregiver. 